\title{
Cross Format Embedding of Metadata in Images Using QR Codes
}

\author{
Athanasios Zigomitros and Constantinos Patsakis \\ Department of Informatics, University of Piraeus
}

\begin{abstract}
Image steganography has various usages due to the recent advances in technology, specially in the field of data communication. Even though steganography has been used so far mostly to secretly embed data, we make use of it for embedding cleartext data, which everyone can has access. This work proposes a new method for cross format metadata embedding in images that is based on QR codes, that seems to have several significant advantages over current method of storing metadata.
\end{abstract}

Keywords: steganography, QR codes, LSB, image metadata.

\section{Introduction}

Steganography has been used for centuries and it is only recently that it has become a standardized, well studied and formalized part of computer science. The main reason for its adoption is its core nature, the fact that it enables us to embed messages in other mediums. The fact that current technology gives us the privilege to communicate using several medias, compared to the past and that it enables others to have access to communication via covert channels, triggers a need for privacy and secrecy.

Of course steganography can have malicious applications, depending on the nature of the content of the message that is embedded in the medium, yet as a knife can be used for good or malicious objectives, steganography has more to offer. In the past years, steganography has been widely used for watermarking and applying DRM in images, audio and video assets, as it doesn't alter the mediums significantly and the quality remains the same.

Image steganography has several techniques, the most famous is LSB embedding, where the data are being stored on the least semantic bits, so that they are not detectable by the eye. Moreover, if the pixels that are used are picked using a good and secure pseudo-random function, then the embedded information remains secure. Other techniques use the frequency domain like DCT and DWT. A good introduction to steganographic techniques is given in [12].

This work tries to make use of QR codes in order to embed image metadata inside them. The reason for doing so is that when changing image format metadata, which in many cases are very useful, are lost. Our approach towards this problem is to alter the image and embed the metadata in the image, so that if lossless image formats are used, then the metadata remain untouched. The 
proposed method provides several advantages that are going to be discussed in the following sections.

\section{QR Codes}

Barcodes are being used for more than three decades in order to help us extract data from databases about the objects to which they are attached. From product prices to blood rhesus and phenotype, barcodes have proved to be very beneficial. Yet, they have an inherent problem due to their structure. Barcodes are not suitable to store information, but only tags about the information that can later be used to identify it using a proper database.

QR codes come to fill in this gap, as they are a generalization of barcodes. These codes can be regarded as the two dimensional version of barcode and have started gaining wider acceptance as an industry standard. The name is from the initials of "Quick Response" and depending on the standard that will be used may contain several thousands bytes of information. Semacode [4, which is very popular as well, is another form of two dimensional barcodes and has been developed to store mainly URLs, thus their structure does not store much information. Both of these codes resemble a crossword puzzle but follow special patterns in order to store the information and be easily read from mobile devices.

Due to the development of smart-phones, QRs have found their way on the market, being used by many applications on iOS and Android mobile platforms. An example of a QR and its anatomy is presented in Figure ?? For extensive information on QR codes and its standards the reader may refer to [3].

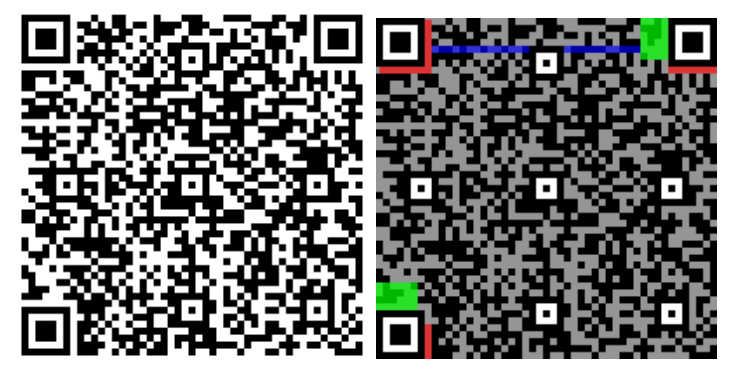

Fig. 1. An example QR code containing as data the text "Cross format embedding of metadata in images using QR codes. Athanasios Zigomitros, Constantinos Patsakis. Department of Informatics, University of Piraeus.". On the left its anatomy is presented, timestamps, version etc.

\section{Our Proposal}

The use of QR in steganography has already been made [5]7], but the applications of the proposed method are wider and more generic. 
The proposed algorithm takes as input the data to be stored in QR form and a random seed $\mathrm{R}$. The random seed is stored in a 32 by 32 square which is stored in the upper left corner of the picture using LSB. The result is that the image is divided as in Figure ??] as an alternative, the first row of the pixels of the image can be used as the random seed. We use the upper square in order to create to more rectangles which will store information like, what type of content is stored, what is the size of the QRs how they are arranged or even the hash of the embedded QRs.

The random seed $\mathrm{R}$ is used in order to initialize a pseudo random permutation $\mathrm{P}$ which will scramble the QR squares in the image. After the scramble, the QRs are embedded in the image by altering the least significant bits of the image. The extraction process is exactly the reverse. On receiving the image, we extract $\mathrm{R}$ and we have the random permutation, thus by using $P^{-1}$ we can find the bits which that are embedded in the QRs.

Both the embedding and extraction processes can be seen in Figure 3 ,

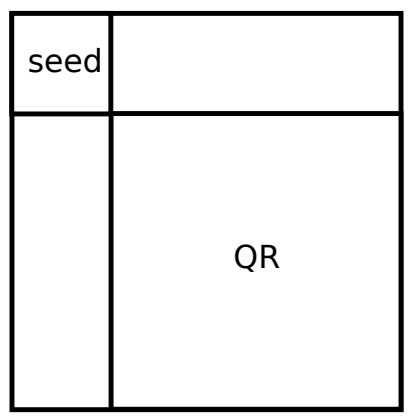

Image

Fig. 2. QR embedding

\section{Results}

The tests that are presented in this work were made with grayscale pictures, but could easily be applied to full color ones. Figure ?? shows several well known "free" images and the image after QR embedding. For the images that we used in our experiments, we made some image measurements and the results can be seen in Table 1. The next two figures, Figure ??] and Figure [??, show two distorted images and the recovered QR. Due to the structure of the QR and the scrambled embedding of it at the image a noise removal filter can improve the quality of the retrieved $\mathrm{QR}$ when the watermarked image was distorted. The QR can also be used as a fragile watermark and in his scrabbled form is possible to show a pattern of distortion in the altered image Figure ?? (b). 

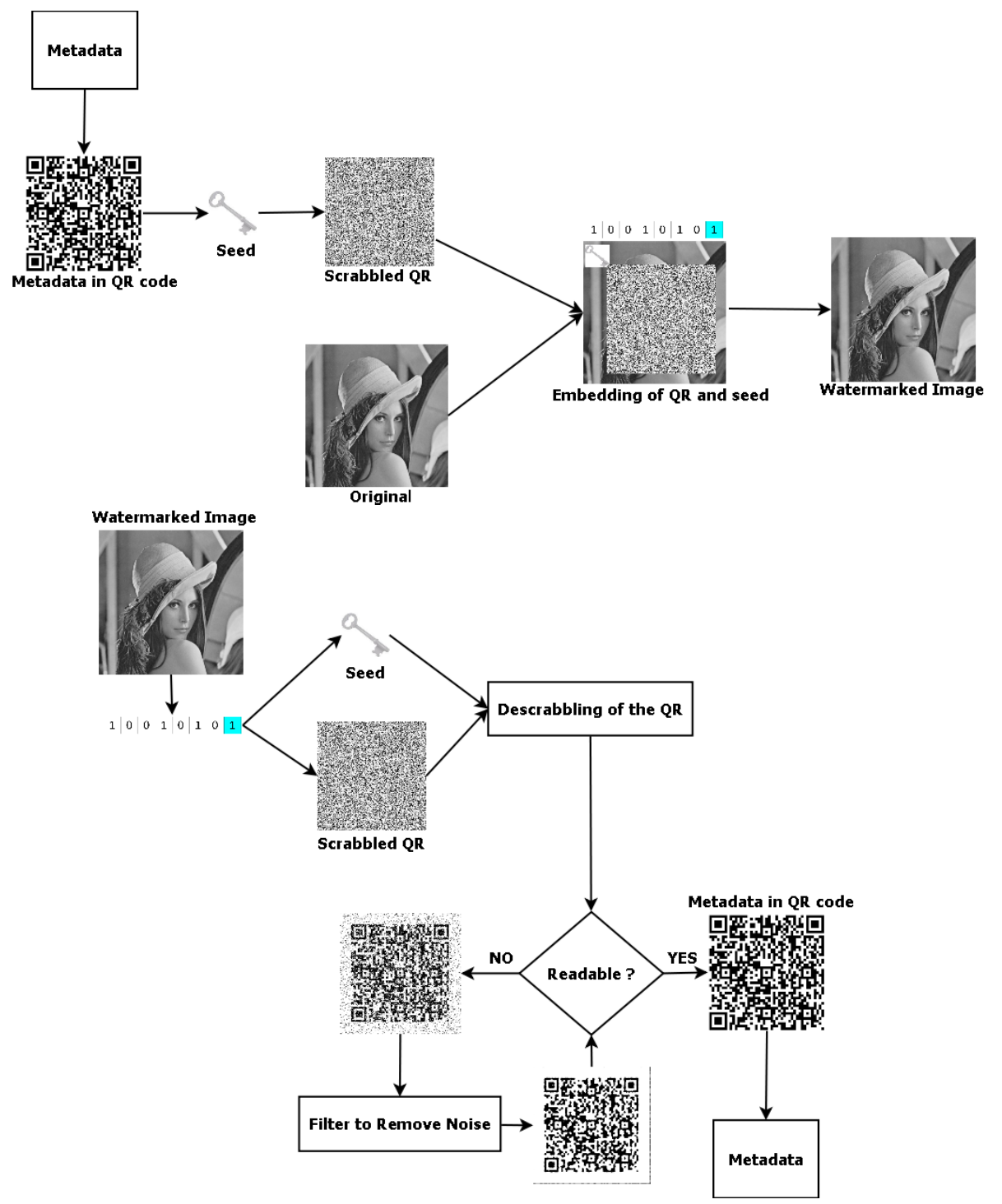

Fig. 3. Embedding and extraction process 

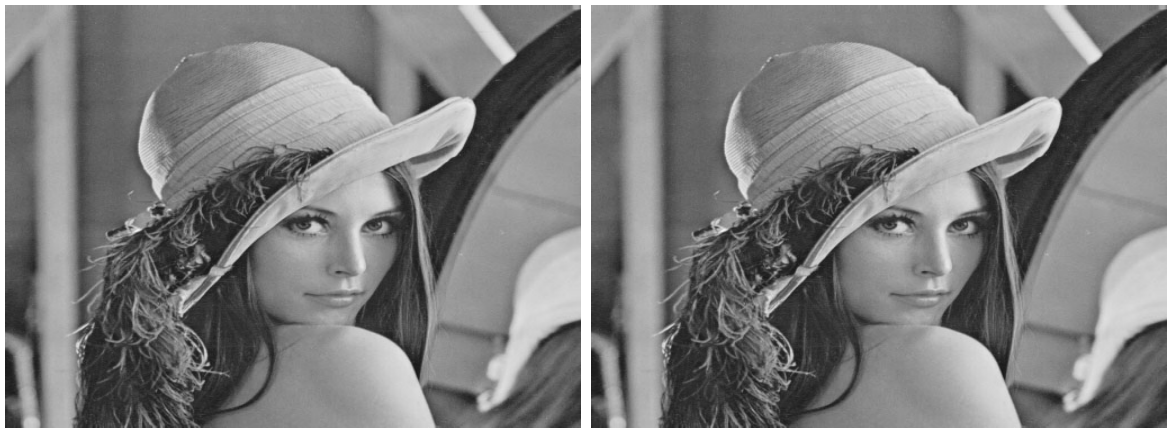

(a) Lena picture and on the right the watermarked version.
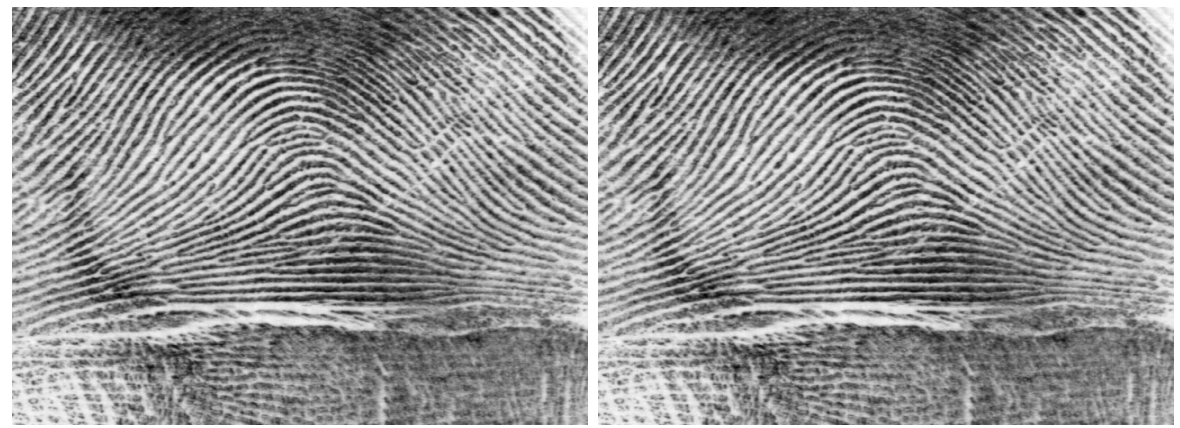

(b) Fingerprint picture and on the right the watermarked version.
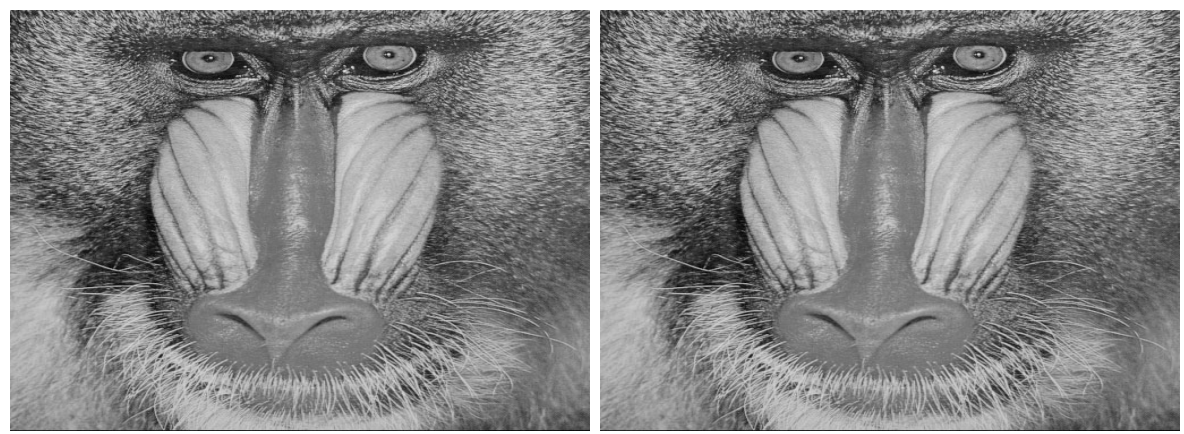

(c) Baboon picture and on the right the watermarked version.

Fig. 4. Image results for some well known pictures 

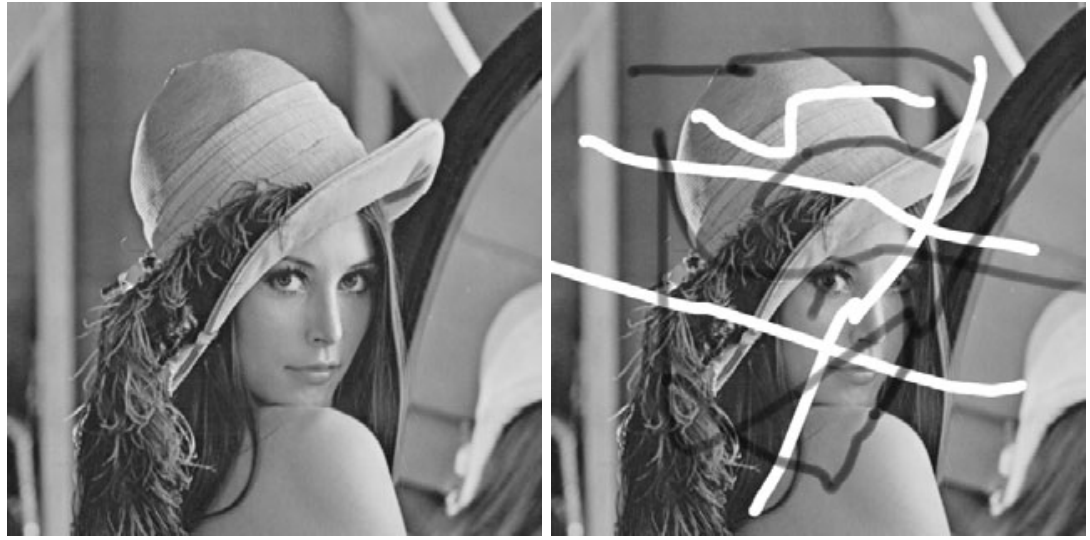

(a) Lena watermarked picture and on the right the distorted version.
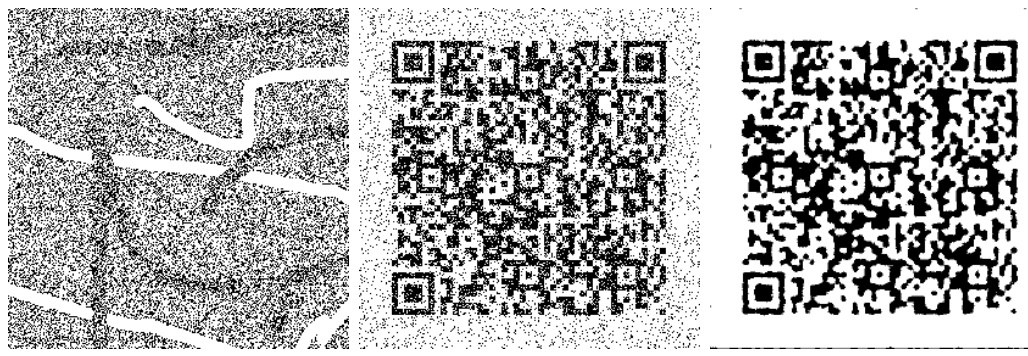

(b) Retrieved Scrambled QR, Retrieved QR data before and after filtering.

Fig. 5. Image results for some well known pictures after embedding and distortion

Table 1. Image measurements

\begin{tabular}{|c|c|}
\hline Measurement of 51 test images & amp; Value \\
\hline \hline Mean of Mean Square Error & amp; 0.1513 \\
\hline Mean of Peak Signal to Noise Ratio & amp; 56.3397 \\
\hline Mean of Normalized Cross-Correlation & amp; 0.9995 \\
\hline Mean of Structural Content & amp; 1.0010 \\
\hline Mean of Average Difference & amp; 0.0563 \\
\hline Mean of Maximum Difference & amp; 1 \\
\hline Mean of Normalized Absolute Error & amp; 0.0016 \\
\hline
\end{tabular}

The measurements were made using the Matlab package Image quality measures, developed by Athi Narayanan. 

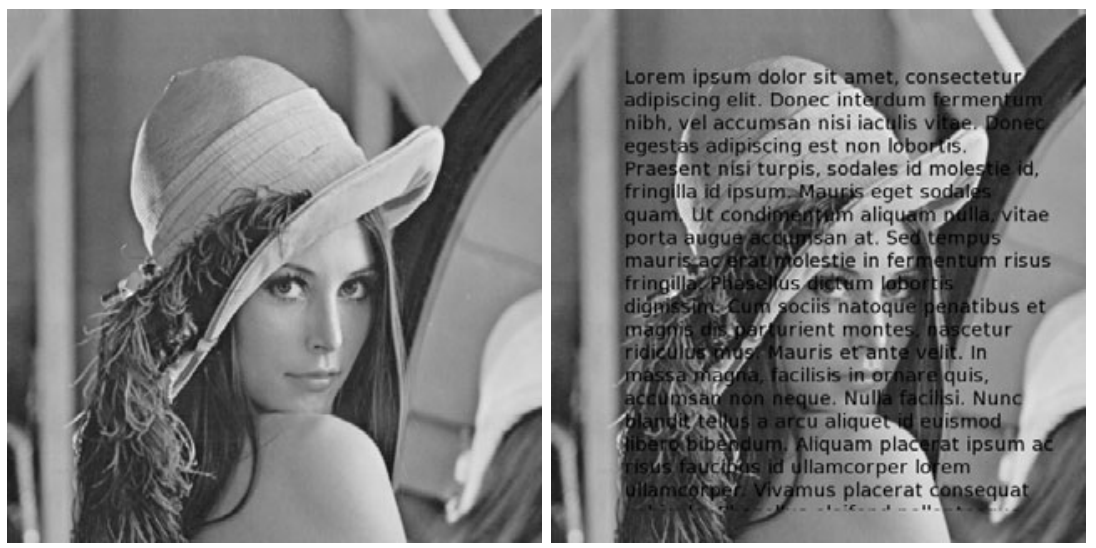

(a) Lena watermarked picture and on the right the distorted version.
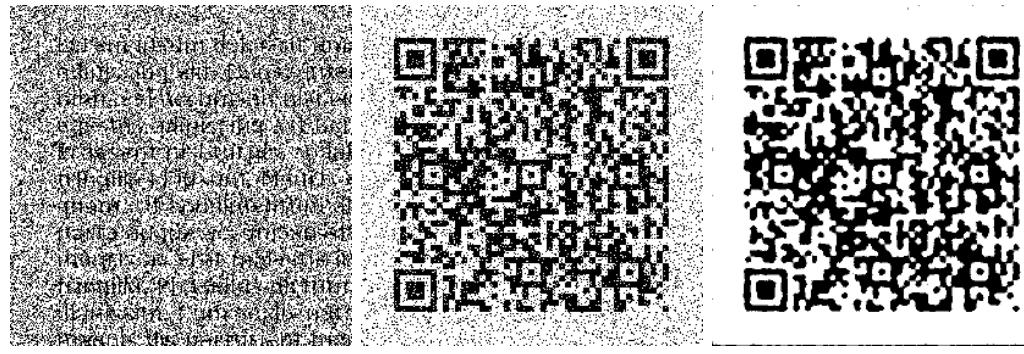

(b) Retrieved Scrambled QR, Retrieved QR data before and after filtering..

Fig. 6. Image results for some well known pictures after embedding and distortion

\section{Applications}

The proposed method has several advantages to offer with its usage. Firstly it enables independent cross format metadata over lossless image formats, thus important data like creator, dates etc can be easily embedded and transmitted without any losses if the image is saved in another format. Moreover, a standardized metadata embedding can prove to be an ideal method for speeding up image searches on the Internet, through search engines.

Secondly, the method can be used to compress usual HTML code. Since the anchor $(<\mathbf{a}\rangle)$ tag in common HTML code is very usual, it can be embedded inside the picture compressing usual HTML code significantly, given the fact that current web pages have re-directions when clicking images. Moreover, the QR could have javascript code embedded, offering fancy image effects when interacting with the photos. Of course this technique will demand more processing from the browser, yet this cost may be overridden by the decrease of traffic load on central servers and routers. 
The proposed method can retrieve data after image cropping and distortion. QRs have already embedded in their structure error detecting and correcting codes that can be used to retrieve image contents if the distortion is not severe. In the case of image cropping, depending on what parts of the image have been left out, embedded data can still be retrieved, as not all of the embedded QRs will have been removed.

By having access to image metadata, it is very easy to make cross site checks for digital rights infringement. In most cases the images have small distortion or they are saved on different image format. Using the proposed metadata embedding techniques, one could detect easier these cases and prove their true origin, yet it is obvious that the method alters any already embedded hidden watermark.

The proposed method could be used in medical images as well, where metadata is an essential need and the alteration of the images must be minimum. For applications of steganography and watermarking and in medical images the reader may refer to [8].

\section{Conclusions}

Currently, the proposed method enables browsers to have access to the image metadata independent of the format, while enabling images to have extra code embedded. On one hand this compresses the data that are transmitted, while on the other hand it makes images "alive".

A possible extension of the presented method would be to enable high definition cameras on mobile device to be able to extract stored information of images, printed or not. The human eye can't read barcodes or QRs so there is no point of seeing it. Of couse the trade off would be the quality loss of the image because the noise that interferes between the image and the camera won't make it readable by the device with the proposed method but a possibly solution to this is to altering bits of higher value or by applying certain filters over specific areas of an image. This would enable cameras to take photos of already content-aware objects and suppling the embedded information.

This method easily can be extended to video files, where one could embed metadata to every frame of the video e.g. subtitles.

\section{References}

1. Wayner, P.: Disappearing Cryptography: Information Hiding: Steganography \& Watermarking, 3rd edn. Morgan Kaufmann, San Francisco (2008)

2. Cox, I., Miller, M., Bloom, J., Fridrich, J., Kalker, T.: Digital Watermarking and Steganography, 2nd edn. Morgan Kaufmann, San Francisco (2007)

3. QR codes, http://www.denso-wave.com/qrcode/

4. Semacode, http://www. semacode.com/

5. Zhang, S., Yoshino, K.: DWT-Based Watermarking Using QR Code. Science Journal of Kanagawa University 19, 3-6 (2008) 
6. Chen, W.-Y., Wang, J.-W.: Nested image steganography scheme using QR-barcode technique. Optical Engineering 48(5), 057004 (2009)

7. Chung, C.-H., Chen, W.-Y., Tu, C.-M.: Image Hidden Technique Using QR-Barcode. In: Fifth International Conference on Intelligent Information Hiding and Multimedia Signal Processing, pp. 522-525 (2009)

8. Coatrieux, C.G., Lecornu, L., Roux, C., Sankur, B.: A Review of Image Watermarking Applications in Healthcare. In: EMBC 2006: IEEE Int. Conference on Engineering in Medicine and Biology, New York (September 2006) 